\title{
Complete genome sequence analysis of the thermoacidophilic verrucomicrobial methanotroph "Candidatus Methylacidiphilum kamchatkense" strain Kam1 and comparison with its closest relatives
}

Thomas Kruse* (D), Chandini Murarilal Ratnadevi, Helge-André Erikstad and Nils-Kåre Birkeland*i)

\begin{abstract}
Background: The candidate genus "Methylacidiphilum" comprises thermoacidophilic aerobic methane oxidizers belonging to the Verrucomicrobia phylum. These are the first described non-proteobacterial aerobic methane oxidizers. The genes $p m o C A B$, encoding the particulate methane monooxygenase do not originate from horizontal gene transfer from proteobacteria. Instead, the "Ca. Methylacidiphilum" and the sister genus "Ca.

Methylacidimicrobium" represent a novel and hitherto understudied evolutionary lineage of aerobic methane oxidizers. Obtaining and comparing the full genome sequences is an important step towards understanding the evolution and physiology of this novel group of organisms.

Results: Here we present the closed genome of "Ca. Methylacidiphilum kamchatkense" strain Kam1 and a comparison with the genomes of its two closest relatives "Ca. Methylacidiphilum fumariolicum" strain SolV and "Ca.

Methylacidiphilum infernorum" strain V4. The genome consists of a single 2,2 Mbp chromosome with 2119 predicted protein coding sequences. Genome analysis showed that the majority of the genes connected with metabolic traits described for one member of "Ca. Methylacidiphilum" is conserved between all three genomes. All three strains encode class I CRISPR-cas systems. The average nucleotide identity between "Ca. M. kamchatkense" strain Kam1 and strains SolV and V4 is $\leq 95 \%$ showing that they should be regarded as separate species. Whole genome comparison revealed a high degree of synteny between the genomes of strains Kam1 and SolV. In contrast, comparison of the genomes of strains Kam1 and V4 revealed a number of rearrangements. There are large differences in the numbers of transposable elements found in the genomes of the three strains with 12, 37 and 80 transposable elements in the genomes of strains Kam1, V4 and SolV respectively. Genomic rearrangements and the activity of transposable elements explain much of the genomic differences between strains. For example, a type $1 \mathrm{~h}$ uptake hydrogenase is conserved between strains Kam1 and SolV but seems to have been lost from strain V4 due to genomic rearrangements.

(Continued on next page)
\end{abstract}

\footnotetext{
* Correspondence: Thomas@theviking.dk; Nils.birkeland@uib.no

Department of Biological Sciences, University of Bergen, P.O. Box 7803, 5020

Bergen, Norway
}

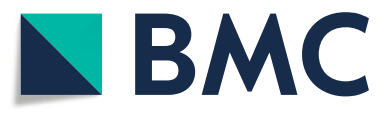

(c) The Author(s). 2019 Open Access This article is distributed under the terms of the Creative Commons Attribution 4.0 International License (http://creativecommons.org/licenses/by/4.0/), which permits unrestricted use, distribution, and reproduction in any medium, provided you give appropriate credit to the original author(s) and the source, provide a link to the Creative Commons license, and indicate if changes were made. The Creative Commons Public Domain Dedication waiver (http://creativecommons.org/publicdomain/zero/1.0/) applies to the data made available in this article, unless otherwise stated. 
(Continued from previous page)

Conclusions: Comparing three closed genomes of "Ca. Methylacidiphilum" spp. has given new insights into the evolution of these organisms and revealed large differences in numbers of transposable elements between strains, the activity of these explains much of the genomic differences between strains.

Keywords: "Ca. Methylacidiphilum kamchatkense" strain Kam1, Full genome, Methane oxidation, Thermoacidophile, Methanotroph

\section{Background}

Interest in methane oxidizing bacteria has been fueled by the fact that methane on one hand; is estimated to be a 25 times stronger greenhouse gas than $\mathrm{CO}_{2}$, seen over a century [1]; on the other hand methane is an inexpensive starting material for biochemical synthesis of high value products [2]. The majority of all described aerobic methane oxidizing bacteria belongs to the alpha- or gamma-proteobacteria. Members of the verrucomicrobia and the intra-aerobic members of the candidate phylum NC10 are notable exceptions [3, 4]. In 2007-8 three groups independently described the isolation of methanotrophic thermoacidophilic verrucomicrobia, strains Kam1, SolV and V4 from acidic geothermal sites in Russia, Italy and New Zealand respectively [5-7]. Based on the low $(<83 \%) 16$ S rRNA gene similarity to other verrucomicrobia it was suggested that they represent a new order, with the proposed order/family/genus names "Candidatus ( $\mathrm{Ca}$.) Methylacidiphilales/Methylacidiphilacea/Methylacidiphilum" [3]. Average nucleotide identity (ANI) comparison showed that strains SolV and V4 constitute two different species with the proposed species names " $\mathrm{Ca}$. Methylacidiphilum fumariolicum" and " $\mathrm{Ca}$. Methylacidiphilum infernorum" respectively. Due to lack of full genome information for strain Kam1, the ANI between this strain and SolV/V4 could not be determined. Despite the fact that the $16 \mathrm{~S}$ rRNA genes of strains Kam1 and SolV are $99.7 \%$ identical, it was proposed that the former also represents a novel species, "Ca. Methylacidiphilum kamchatkense" [3]. For the remainder of this paper we will predominately use the strain designations Kam1, SolV and V4 to refer to the different representatives. Additional acidophilic, thermophilic and mesophilic, verrucomicrobial methanotrophs have been isolated from acidic environments demonstrating that these may be widespread $[8,9]$.

Members of the "Ca. Methylacidiphilaceae" are gram negative, non-motile moderate thermoacidophiles with a growth optimum at $55-60{ }^{\circ} \mathrm{C}$ and capable of growing at a wide $\mathrm{pH}$ range of 0.8 to 6 . Their genomes do not encode a soluble methane monooxygenase (sMMO), but contains three $p m o C A B$ operons, coding for the particulate methane monooxygenase (pMMO). Strain Kam1 encodes an additional unique and truncated pmoCA cluster that is not present in strains SolV and V4 $[3,10]$.
The pmo genes from "Ca. Methylacidiphilum spp." form a distinct phylogenetic group, separate from their proteobacterial counterparts, showing that they do not originate from a recent horizontal gene transfer [3].

The "Ca. Methylacidiphilaceae" possess a range of traits clearly separating them from their proteobacterial counterparts. They are the most acidophilic methanotrophs described and grow autotrophically fixing $\mathrm{CO}_{2}$ via the Calvin Benson Bassham (CBB) cycle. Unlike proteobacterial methanotrophs that assimilate carbon via either; the RuMP cycle (group I) or the serine cycle (Group II) $[2,11]$. Finally their methanol dehydrogenases are of the XoxF type containing lanthanides, a group of rare earth elements, instead of calcium in the active site [12]. Closed genomes are available for strains SolV and V4, whereas a multi-contig draft genome has been published for strain Kam1 [13-15]. Genome analysis has given a deeper understanding of the metabolism of strains SolV and V4. For example, a number of genes predicted to encode hydrogenases were observed in the genomes of strains SolV and V4 [13, 14]. This led to the speculation that they might be capable of autotrophic growth on $\mathrm{H}_{2}, \mathrm{O}_{2}$ and $\mathrm{CO}_{2}$, a metabolic trait that later has been verified experimentally for strain SolV and the recently described " $\mathrm{Ca}$. Methylacidiphilum sp. strain RTK17.1" [9, 16].

Comparison of the closed genomes of strains SolV and $\mathrm{V} 4$, revealed large numbers of genomic rearrangements, hampering detailed comparison of genome architecture. Comparisons of the protein encoding genes showed that $64,3 \%$ of the protein encoding genes from SolV have more than $50 \%$ amino acid identity to genes from strain V4 [14].

We here present the closed genome of " $\mathrm{Ca}$. Methylacidiphilum kamchatkense" Kam1, and compare it with the two previously published genomes from the " $\mathrm{Ca}$. Methylacidiphilum" genus. We use genome comparisons as backbone for discussion of phylogeny and genome architecture of strains Kam1, SolV and V4. Finally, we link genome analysis with metabolic and physiological traits reported in the literature.

\section{Results}

We employed PacBio RS technology in order to obtain the complete genome sequence of " $\mathrm{Ca}$. Methylacidiphilum 
kamchatkense" strain Kam1. After quality, checks of the reads we ended with 94,711 reads with an average read length of 14,734 and N50 read length of 21,352 bp with an average coverage of 489 .

After assembly and manual trimming of overlapping ends, we obtained the full genome of strain Kam1. The closed genome of Kam1 consists of a single circular 2, 202,032 bp chromosome, which is approximately 85 and $275 \mathrm{kbp}$ less than the genomes of V4 and SolV, respectively (Table 1 ). We did not identify any extrachromosomal genetic elements in strain Kam1. The annotation identified 2119 coding sequences hereof 1589 with a predicted function (Table 1). The genome encodes a single $16 \mathrm{~S}$ rRNA operon and four pmo $A$ genes, the latter feature distinguishes Kam1 from SolV and V4 that harbor three pmoA genes (Table 1) [13-15]. We identified 17 CRISPR spacer sequences but no integrated phages in the genome of strain Kam1 (Table 1).

Until now, it has been unclear if strains SolV and Kam1 constitute two different species or not [3].

The average nucleotide identity (ANI) is a fast and easily reproducibly in-silico alternative to experimental DNADNA hybridization. An ANI value of $95 \%$ corresponds to the $70 \%$ species cutoff used for DNA-DNA hybridization [18]. Previously, a subsample of randomly chosen genes from SolV was used to calculate an ANI value of $73 \%$ for strains SolV and V4, clearly showing that they constitute two different species, "Ca. M. fumariolicum" and "Ca. M. infernorum", respectively [3]. We used the closed genomes of strains Kam1, SolV and V4 to calculate the ANI between the three strains (Additional file 1: Table S1). The obtained ANI values between strains Kam1, SolV and V4 are all below 95\%, therefore, they should be considered as type strains of three different species, "Ca. M. kamchatkense", "Ca. M. fumariolicum" and "Ca. M. infernorum" respectively (Additional file 1: Table S1).

We then used the annotated genome for a detailed analysis, linking genomic data with metabolic and physiological traits reported in the literature. This analysis includes a comparison of the genomes of strains Kam1, SolV and V4. The result of which will be presented in detail in the discussion section.

\section{Discussion}

\section{Mobile elements}

Dot plot comparison of the three genomes revealed a very high degree of conservation of the genome architecture between strains Kam1 and SolV (Additional file 1: Figure S1-A), whereas several rearrangements and inversions of large genomic regions were identified when comparing strains Kam1 or SolV with V4 (Additional file 1: Figure S1-B-C).

The large number of rearrangements and the observation of several gaps in whole genome alignments of strains Kam1, SolV and V4, prompted us to investigate and compare the presence and abundance of mobile elements.

Table 1 Genomic properties of the three "Ca. Methylacidiphilum" species for which a closed genome is available

\begin{tabular}{|c|c|c|c|}
\hline & $\mathrm{Kam} 1^{\mathrm{a}}$ & SolV $V^{b}$ & $\mathrm{~V} 4^{\mathrm{c}}$ \\
\hline Size (bp) & $2,202,032$ & $2,476,671$ & $2,287,145$ \\
\hline Contigs & 1 & 1 & 1 \\
\hline GC \% & 40.34 & 41.48 & 45.48 \\
\hline RNAs & 53 & 49 & 52 \\
\hline CDS & 2119 & 2741 & 2473 \\
\hline $16 S$ & 1 & 1 & 1 \\
\hline pmoAs & 4 & 3 & 3 \\
\hline CRISPR spacers & 17 & 23 & 20 \\
\hline Phages & 0 & 0 & 0 \\
\hline Integrated plasmids & 1 & 1 & 1 \\
\hline Transposable elements & 12 & 80 & 37 \\
\hline Genomic islands & 2 & 22 & 9 \\
\hline Locus tag prefix & Ga0255985_11 ${ }^{\mathrm{e}} ; \mathrm{kam} 1 \_^{\mathrm{f}}$ & 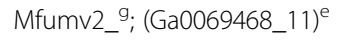 & Minf__ \\
\hline IMG genome $I^{d}$ & $2,770,939,480$ & $2,630,968,640$ & $642,555,138$ \\
\hline Genbank accession & СР037899 & PRJEB6910 & CP000975 \\
\hline
\end{tabular}

a"Ca. Methylacidiphilum kamchatkense" Kam1, this study

b"Ca. Methylacidiphilum fumariolicum" SolV, [14]

c"Ca. Methylacidiphilum infernorum" V4, [13]

dintegrated Microbial Genomes and Microbiomes [17]

e Locus tag prefixes from Integrated Microbial Genomes and Microbiomes IMG/ER [17]

fLocus tag prefixes from National Center for Biotechnology Information (NCBI)

${ }^{g}$ Locus tag prefixes and annotation as given by Mohammadi and colleagues 2017 [16] 
The presence of $\mathrm{a} \approx 45 \mathrm{kbp}$ putative integrative plasmid has been reported in the genome of strain V4 [13]. Whole genome alignments revealed that this putative integrative plasmid is present in both strain Kam1 and SolV, although some insertions or deletions has occured. The putative integrated plasmid is located in the same region of the genome in all three strains, showing that it was present in their last common ancestor. No prophages were found in any of the three genomes.

All three strains encode a class I, type III CRISPR-cas adaptive immune system, involved in protecting against phages and plasmids $[19,20]$. The three genomes encode 17-23 CRISPR spacer sequences, all spacers are species specific but in a few cases, two identical copies are present within the same strain (Table 1, Additional file 2). The spacers and CRISPR associated genes are found in different regions in all three genomes. The Cas2 proteins from strains Kam1 and SolV are 81\% identical and the gene order and content of the CRISPR regions are highly similar. In contrast, the two Cas 2 proteins from strain $\mathrm{V} 4$ is only $14-16 \%$ identical to their $\mathrm{Kam} 1 / \mathrm{SolV}$ counterparts and the entire CRISPR region differs from Kam1/SolV (Additional file 1: Figure S2). The cas1 gene from strain V4 is truncated; it is therefore likely that the CRISPR system of this strain is non-functional [13]. Whereas the CRISPR systems of strains Kam1 and SolV appears to be functional. The presence of different CRISPR systems in the genomes of strains Kam1/SolV and V4, suggests that either one or both has been acquired horizontally from different donors.

The genome of strain Kam1 encodes only two predicted genomic islands (GI)s with a size of 17 (GI-I) and $4,8 \mathrm{~kb}$ (GI-II) respectively, in contrast to the 22 and 9 predicted GIs with a total size of $205.5 \mathrm{~kb}$ and $111.4 \mathrm{~kb}$ found in the genomes of strains SolV and V4, respectively, (Table 1; Fig. 1, Additional file 1: Figures S3-S4). GI-I encodes 18 predicted genes, whereof 14 is without a predicted function; the majority of the genes with a predicted function encodes DNA modification systems. GI-II encodes four predicted genes, whereof two is without a predicted function (Additional file 1: Table S2). GI-I is not present in the genomes of strains SolV and V4, whereas GI-II is present

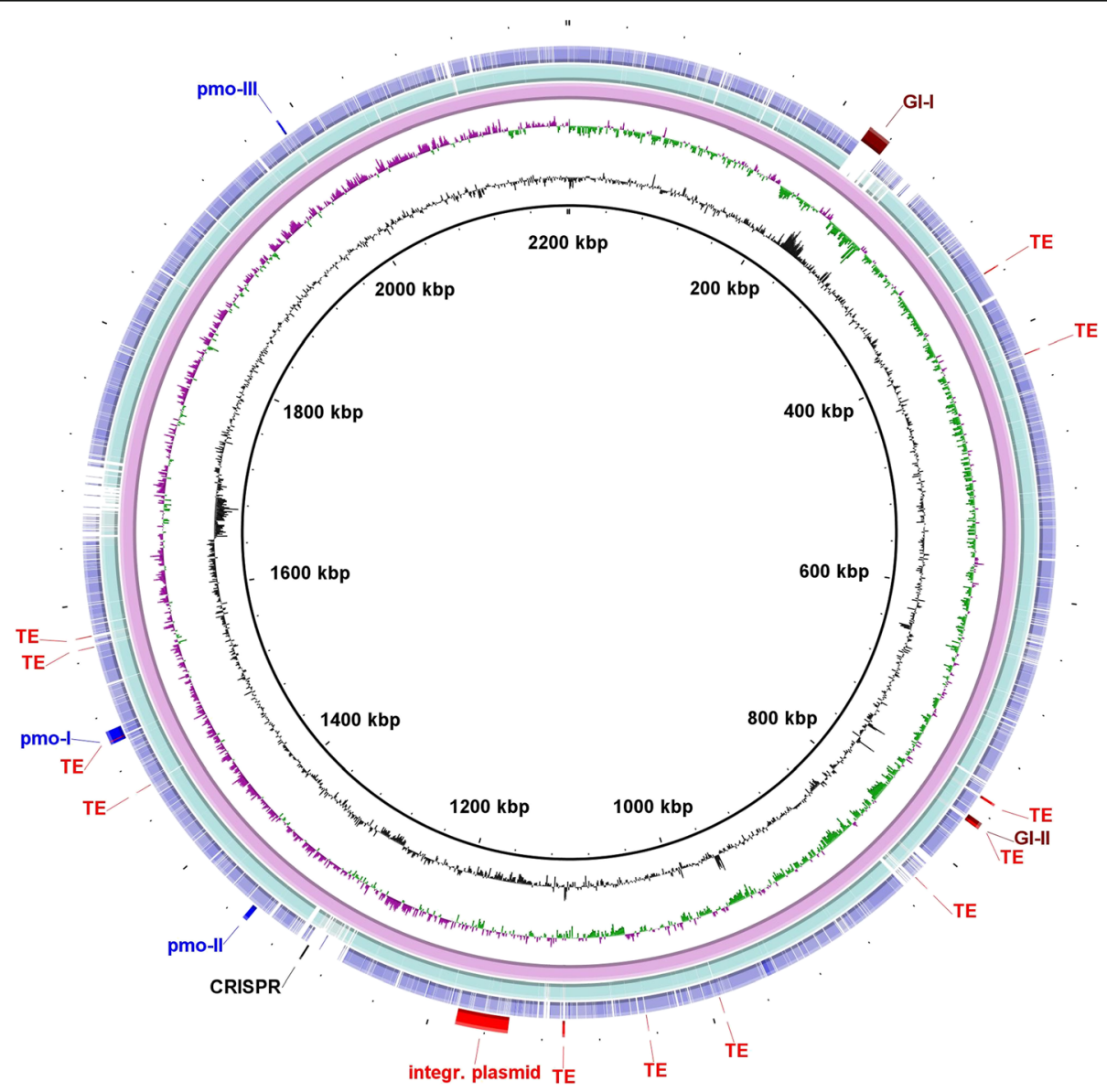

Fig. 1 Circular representation of the genome of "Ca. Methylacidiphilum kamchatkense" Kam1. Rings from inside to outside: 1) GC content (black); 2) GC skew (-/+ purple/green); 3) strain Kam1; 4) strain SolV; 5) strain V4; 6) Selected genomic traits of strain Kam1, for exact genomic coordinates see (Additional file 2). Gl: Genomic island; TE: Transposable element; Pmo: pmo cluster, see also Fig. 2 
in both strains Kam1, SolV, and partially in V4 (Fig. 1 ). Two out of five analyzed genes from GI-I, gave blastn hits to genomes of non-verrucomicrobial thermophiles and one to a plasmid from a mesophile, whereas the last two sequences, did not resemble any sequences in the databases. The four predicted genes from GI-II gave blastn hits to strains SolV and $\mathrm{V} 4$ and in two instances to other thermophilic bacteria (Additional file 1: Table S2). Similar observations were made for the GIs from strains SolV and V4 (data not shown). Since the majority of the genes found in GI-I and II is without a predicted function, it is not possible to say whether the presence of homologs in other unrelated thermophiles or thermoacidophiles means that they encode beneficial traits for survival in this niche or simply reflects horizontal gene transfer between organisms found in the same environment.

Transposons and other insertion sequences, from here on collectively referred to as transposable elements (TE), are small genomic elements capable of moving within or between genomes. Movement can be facilitated by genes such as transposases, encoded by the TE itself or by encoded sequences recognized by transposases encoded by other TEs, integration into f. ex. conjugative plasmids allows horizontal gene transfer of TEs (for review see [21]). The presence of TEs can have profound effects on bacterial genomes, by disrupting genes, altering gene expression or lead to deletions and rearrangements within the genome $[21,22]$.

We searched the genomes of strains Kam1, SolV and V4, available at the Joint genome institutes IMG/ER server for annotated transposases using the search term "transpos" [17]. We found four transposases annotated in the previously published draft genome of strain Kam1, whereas the closed genomes of strains Kam1, SolV and V4 encode 3, 48 and 23 annotated transposases, respectively [13-16].

In total we identified 12 TEs in the closed genome of strain Kam1 and a large number TEs in the genomes of strains SolV and V4 (Table 1 and Additional file 2). The TEs from all three "Ca. Methylacidiphilum" strains are generally located in regions with either no or low homology to the other two genomes, indicating that these are either species specific or in various stages of decay (Fig. 1 Additional file 1: Figures S3-S4).

It is likely that TEs have had a large impact on the evolution of "Ca. Methylachidiphilum". Examples of this is the presence of TEs integrated into pmo cluster I, between $p m o B_{2}$ and $p m o C_{5}$ (Fig. 2), indications that movement of TEs have led to the loss of $p_{m o C A}$ from strains SolV and V4 and the loss of the type $1 \mathrm{~h} \mathrm{NiFe} \mathrm{hy-}$ drogenase from the genome of strain V4, as will be discussed below.

\section{Methane metabolism}

Genome analysis confirmed the absence of genes encoding sMMO. The genome of Kam1 encodes three pmoCAB operons, a truncated pmoCA operon and a separate $p m o C$ gene, as reported previously (Fig. 2) [3, 10]. The methane monooxygenases from "Ca. Methylacidiphilum spp." and other verrucomicrobia constitute a distinct group, clearly separated from their proteobacterial counterparts. The $p m o A$ gene is present in all known aerobic methanotrophs, and is commonly used as marker gene, for detection of potential for methanotrophy in environmental samples. The primers used for detection of $p m o A$ from proteobacteria, do not amplify $p m o A$ genes from verrucomicrobia nor from the intra-aerobic members of the candidate phylum NC10 under standard PCR conditions. This may have led to an underestimation of the abundance and diversity of methanotrophs in some environments [3, 23].

Recently, a primer pair amplifying the intergenic region between $p m o A$ and $p m o B$ has been reported to target both $p m o C A$ from proteobacteria and $p m o C A_{1-2}$, but not $\mathrm{pmoCA}_{3}$, from strain SolV [24].

The genome of strain Kam1 encodes three pmo gene clusters (I-III, Fig. 2). I) Organized as one large cluster encoding two pmoCAB operons in tandem and a downstream orphan $p m o C$. II) Encoding a single pmoCAB operon and finally III) encoding a pmoCA operon, unique for strain Kam1, where $\mathrm{pmoC}_{4}$ are $\mathrm{N}$-terminally truncated (Fig. 2, Table 2. Additional file 1: Table S3) [10]. Changing a single base at position $1,986,490$, would change an ATT to an ATG start codon, on the reverse

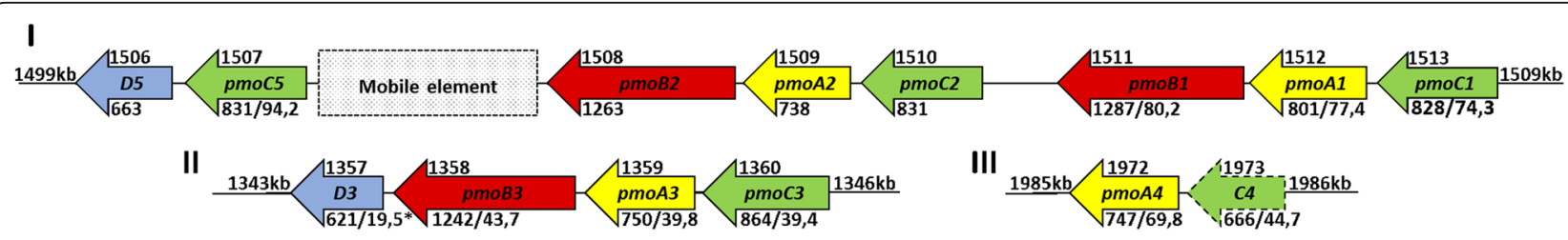

Fig. 2 Organization of pmo cluster I-III from "Ca. Methylacidiphilum kamchatkense" Kam1. Numbers at the end of lines indicate location in the genome. Numbers on top of arrows indicate locus tags without prefix, (see Table 1 for prefixes), numbers under arrows indicate gene size in bp / $\%$ amino acid identity to the $\mathrm{pmo}_{2}$ homolog. ${ }^{*}$ amino acid identity to $\mathrm{PmoD}_{5}$. Arrow surrounded by a dashed line indicates a truncated and thus likely non functional gene 
Table 2 Presence $(\sqrt{ })$, absence (---) or number of genes or groups of genes encoding selected metabolic traits

\begin{tabular}{llll}
\hline & Kam1 & SolV & V4 \\
\hline pmo clusters & 3 & 2 & 2 \\
Uptake hydrogenase, NiFe, type 1h & $\sqrt{ }$ & $\sqrt{ }$ & - \\
Uptake hydrogenase, NiFe, type 1d & $\sqrt{ }$ & $\sqrt{ }$ & $\sqrt{ }$ \\
Solouble hydrogenase, NiFe, type 3b & $\sqrt{ }$ & $\sqrt{ }$ & $\sqrt{ }$ \\
Calvin Benson Bassham cycle & $\sqrt{ }$ & $\sqrt{ }$ & $\sqrt{ }$ \\
MoFe-Nitrogenase & $\sqrt{ }$ & $\sqrt{ }$ & $\sqrt{ }$ \\
Alternative MQ synt. Pathway & $\sqrt{ }$ & $\sqrt{ }$ & $\sqrt{ }$ \\
Resp. Complex I, 14 subunits & $\sqrt{ }$ & $\sqrt{ }$ & $\sqrt{ }$ \\
Resp. Complex II & $\sqrt{ }$ & $\sqrt{ }$ & $\sqrt{ }$ \\
Complex III, bc1 & - & - & - \\
Alternative complex III & $\sqrt{ }$ & $\sqrt{ }$ & $\sqrt{ }$ \\
Resp. Complex IV CBB3 type & $\sqrt{ }$ & $\sqrt{ }$ & $\sqrt{ }$ \\
Resp complex V, ATP synthase & 2 & 2 & 2 \\
Ech hydrogenase related (Ehr) complex & $\sqrt{ }$ & $\sqrt{ }$ & $\sqrt{ }$ \\
Heavy metal efflux pumps & 8 & 8 & 9
\end{tabular}

Strain designations are given on top of columns. For full species, names see Table 1

strand, restoring a full-length $p m o C_{4}$ with a size of 831 bp (data not shown).

The majority of the organisms encoding pmoCAB, also encodes a membrane protein PmoD $[25,26]$. The physiological role of PmoD, and the closely related AmoD, is still not understood. Their encoding genes are usually found as part of pmo/amoCAB operons, but homologs next to $\mathrm{Cu}$ resistance genes have also been reported [25]. A role as a copper chaperone has been proposed [25, 27]. This speculation is supported by expression data from Methylococcus capsulatus Bath grown in the presence or absence of copper. The expression of a pmoD homologue, MCA2130, located next to a multicopper oxidase family protein was down-regulated in the absence of copper, whereas another $p m o D$ homologue, MCA2170b, located next to a $\operatorname{cop} C$ gene, was upregulated [28]. This is consistent with the speculation that CopC has a role in copper uptake, in organisms possessing the copper dependent particulate methane monooxygease [29]. A recent study demonstrated that PmoD is a membrane bound copper binding protein. Furthermore, knocking out $p m o D$ in Methylosinus trichosporium OB3b resulted in inability to grow using the Copper dependent pMMO, whereas growth using the copper independent sMMO was unaffected [26].

Careful inspection of the genome revealed the presence of a $p m o D$ homologue located directly downstream of $p m o C_{5}$. A blastP search against the entire genome using the sequence of this $P m o D_{5}$ as a query revealed a total of four pmoD homologs in the genome of strain Kam1. Two of these are part of pmo clusters I or II, one is located next to a gene annotated as multicopper oxidase, and one is located in close proximity to a CRISPR region (Fig. 2. Additional file 1: Table S4). All four pmoD homologs encode a single $C$ terminal transmembrane helix, and two out of four encode a signal sequence (Additional file 1: Table S4). We found homologs of all four pmoDs in both strains SolV and V4, although they appear to be slightly less conserved between the three strains than the other pmo genes (Additional file 1: Tables S5-A-D).

The verrucomicrobial $p m o B$ genes do not encode the conserved Copper binding sites found in all other PmoBs [3, 30]. Untill recently it was believed that the active site of pMMO is located in the PmoB subunit [31]. However, the authors of a recent paper found that the active site isn't located in PmoB. In the same paper they found evidence that a predicted zinc/copper binding site (DxxxH $\left.\left(\mathrm{x}_{12}\right) \mathrm{H}\right)$ in PmoC, binds copper, the authors speculated that this may be the active site [32]. Interestingly the $\left(\mathrm{DxxxH}\left(\mathrm{x}_{12}\right) \mathrm{H}\right)$ motif is conserved in all PmoCs from strains Kam1, SolV and V4 (Fig. 3).

The conservation of the copper binding $\left(\mathrm{DxxxH}\left(\mathrm{x}_{12}\right) \mathrm{H}\right)$ motif in all PmoCs; the identification of $p m o D$ homologs as part of the pmo gene clusters in all three " $\mathrm{Ca}$. Methylacidiphilum spp." strains and the observation that growth of strain Kam1 ceased after two transfers in copper free medium. Strongly suggests that the pMMOs of strain Kam1 and likely other " $\mathrm{Ca}$. Methylacidiphilum" strains are copper dependent.

The presence of multiple copies of the pmo genes in strain Kam1, and other methylacidiphila, may be the result of gene duplications. In support of this speculation, the pmo genes located in close proximity of each other are more similar than homologs located more distantly in the genome. Exemplified by the $p m o C s$ from strain Kam1 where the co-located $p m o C_{1}, C_{2}$ and $C_{5}$ are 74$94 \%$ identical to each other but only $39-59 \%$ identical to pmo $_{3}$ and $C_{4}$, that are located elsewhere in the genome (Additional file 1: Table S5-C).

It has been shown that $p m o C A B_{1-3}$ from strains Kam1, SolV and V4 are highly conserved between strains and under intense purifying selection, suggesting that they have evolved to have distinct roles under different conditions $[3,10]$. Furthermore, $p m o C A B_{2}$ is the highest expressed methane monooxygenase under non-limiting growth conditions in both strains Kam1 and SolV [10, 33]. Whereas transcriptomics analysis of strain SolV showed a shift from $p m o C A B_{2}$ to $p m o C A B_{1}$ under oxygen limiting growth conditions [33].

Genome alignments of strains Kam1, SolV and V4 showed that pmo cluster I is located in the same part of the genome in all three strains, although a number of transposable elements are found within and surrounding 


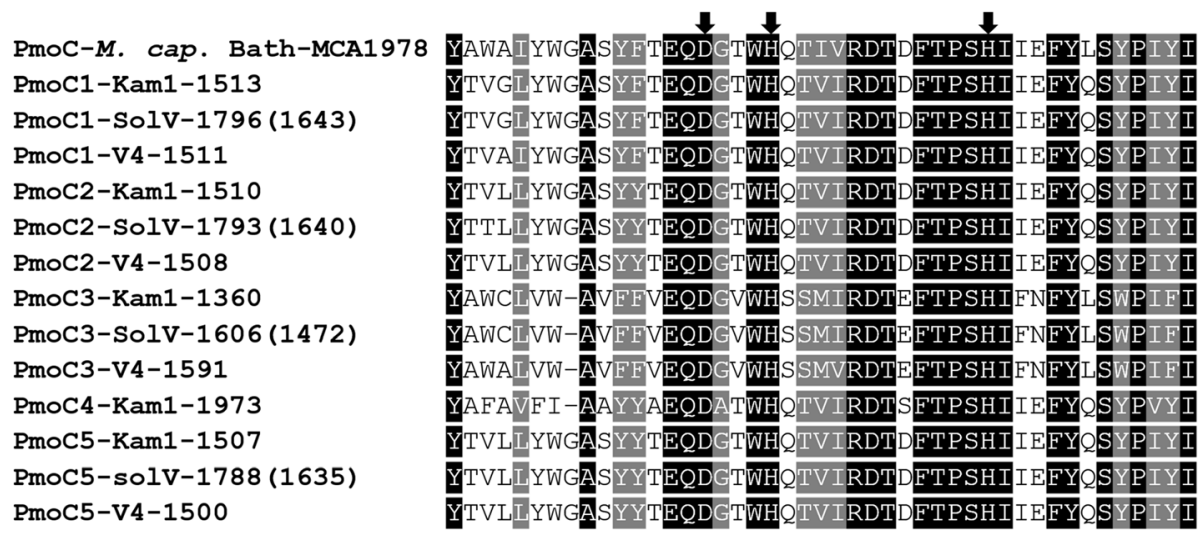

Fig. 3 Alignment of the PmoCs from strains Kam1, SolV and V4 with PmoC from Methylococcus capsulatus strain Bath. PmoC number and strain designations are given followed by locus tags without prefix, (see Table 1 for prefixes). The conserved copper binding motif $\left(D x \times x H\left(x_{12}\right) H\right)$ is indicated with black arrows. For strain SolV locus tags without or between brackets refers to the annotation following Mohammadi and colleagues 2017 [16] or Integrated Microbial genomes and Microbiomes IMG/ER [17] respectively, see also (Table 1)

this cluster (Additional file 1: Figure S5). Pmo cluster II, encoding $p m o C A B D_{3}$, is also located in the same part of the genomes of strains Kam1 and SolV, but is integrated in another part of the genome of strain V4, showing that the whole region has moved within the genome. Full genome alignment revealed large numbers of genomic rearrangements on both sides of $p m o C A B D_{3}$ (Additional file 1: Figure S6). The genome of strain Kam1 encodes pmo cluster III encoding $\mathrm{pmoCA}_{4}$, that are not found in the genomes of strains SolV and V4 [3].

Interestingly we found that $\mathrm{pmoCA}_{4}$, has been replaced by transposable elements in strains SolV and V4. In strain SolV we found fragments of $\mathrm{pmoC}_{4}$ at the $5^{\prime}$ and $3^{\prime}$ end of the inserted transposable element, showing that pmo cluster III was present in the last common ancestor of strain Kam1 and SolV (Additional file 1: Figure S7).

In methanotrophs the methanol produced by the pMMOs oxidation of methane is subsequently oxidized by a methanol dehydrogenase (MDH). The genome of Kam1 does not encode a calcium dependent MDH of the MxaF-type, instead it encodes a XoxF-type, lanthanide dependent $\mathrm{MDH}$. The encoding gene is part of a small operon xoxFJG:J that is also found in the genomes of strains SolV and V4 (Additional file 1: Table S3) [13, 14]. In brief, $x o x F$, encodes the lanthanide dependent MDH. xoxJ, encodes a protein speculated to have a role in binding the MDH to pMMO. xoxG:J, encodes a XoxG and XoxJ fusion protein. The XoxG part is a cytochrome $\mathrm{c}$, that acts as electron acceptor for the $\mathrm{MDH}$, the XoxJ part is truncated compared to the non-fused XoxJ, and has been speculated to have a role in binding XoxG:J to the MDH [12, 34]. Both $\mathrm{MDHs}$ of the MxaF and XoxF-type requires co-factor PQQ [12]. The genomes of all three strains encodes genes, pqqABCDEFG, for biosynthesis of PQQ, these are organized as a $p q q A B C D E$ cluster with $p q q F$ and $p q q G$ located elsewhere in the genomes (Additional file 1: Table S3). MDHs catalyzes the oxidation of methanol to formaldehyde, that then can be further oxidized to $\mathrm{CO}_{2}$ via formate or assimilated into biomass. Formaldehyde is not assimilated by methanotrophic verrucomicrobia, as will be discussed in the "biomass generation" section $[8,11]$. It has been demonstrated that MDH from SolV oxidizes methanol and formaldehyde with the same maximum rate. Leading to the speculation that methanol is oxidized directly to formate [12]. The formate is then further oxidized to $\mathrm{CO}_{2}$ by a NAD dependent formate dehydrogenase (Fig. 4) (Additional file 1: Table S3). In line with this speculation, the genomes of strains Kam1, SolV and V4 do not encode the tetrahydrofolate, tetrahydromethanopterin or the glutathione pathway for formaldehyde oxidation [13, 14, 35].

\section{Biomass generation}

Traditionally methanotrophs have been divided into two groups based on how they assimilate formaldehyde. Group one uses the RuMP pathway, whereas group two uses the Serine pathway [2]. Our analysis showed that strain Kam1, like strains SolV and V4, encodes incomplete RuMP and serine pathways. Genes encoding hexulose-6-phosphate synthase and 6-phospho 3-hexuloisomerase are lacking from the RuMP pathway, and genes encoding Malyl coenzyme A lyase and Glycerate kinase from the serine pathway. Instead it, like strains SolV and V4, encodes a full Calvin Benson Bassham pathway for $\mathrm{CO}_{2}$ fixation (Table 2, Additional file 1: Table S6) [3, 13, 14]. Khadem and colleagues demonstrated that strain SolV obtains carbon by fixing $\mathrm{CO}_{2}$ via the $\mathrm{CBB}$ pathway rather than fixing formaldehyde as conventional methanotrophs [11]. Similar observations has been done for three mesophilic 


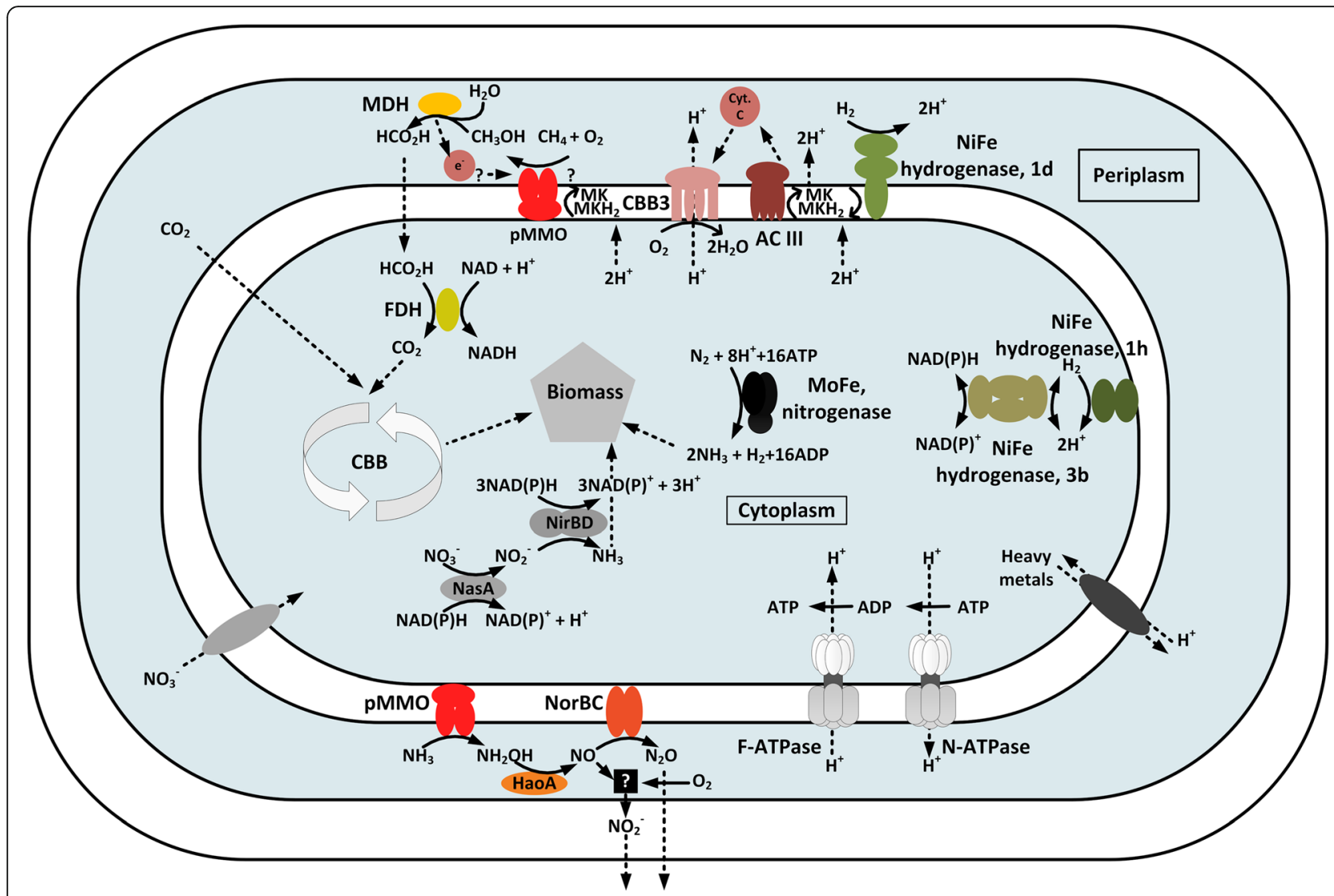

Fig. 4 Schematic representation of "Ca. Methylacidiphilum kamchatkense" Kam1 showing selected metabolic traits. MDH: methanol dehydrogenase; pMMO: particulate methane monooxygenase; CBB3: cbb3 type cytochrome oxidase; AC III: alternative complex III; MK: menaquinone; FDH: formate dehydrogenase; CBB: Calvin Benson Bassham cycle; NasA: assimilatory nitrate reductase; nirBD: nitrite reductase; HaoA: hydroxylamine oxidase; NorBC: nitric oxide reductase; Black box with a question mark: NO oxidation, either nonenzymatic or catalyzed by a yet unidentified NO oxidase

methanotrophic strains from the closely related methylacidimicrobium genus [8]. Most likely this is also the case for strains Kam1 and V4, and possibly a common trait of methanotrophic verrucomicrobia.

We found large numbers of genes associated with glycogen metabolism, in the genome of strain Kam1, all with homologs in the genomes of strains SolV and V4 (Additional file 1: Table S7). Strain SolV has been shown to use glycogen stored in cytoplasmic glycogen vesicles as carbon and energy storage [36]. Similar vesicular structures were observed in strains Kam1 and V4, it is thus plausible that all three strains use glycogen as carbon and energy storage [3, 7]. Some methanotrophs use poly- $\beta$-hydroxybutyrate as carbon and energy storage [37]. We did not identify key genes, phbABC, from the poly- $\beta$-hydroxybutyrate synthesis pathway in the genome of strain Kam1. These genes are also not present in the genomes of strains SolV or V4 [13, 36].

\section{Hydrogenases}

Recently it was demonstrated that strain SolV is capable of autotrophic growth on $\mathrm{H}_{2}, \mathrm{O}_{2}$ and $\mathrm{CO}_{2}$ [16].
Similarly, it has been shown that " $\mathrm{Ca}$. Methylacidiphilum sp." strain RTK17.1, that is closely related to strain V4, employs a mixotrophic lifestyle, oxidizing methane and hydrogen simultaneously [9].

The genome of strain Kam1 encodes three NiFe hydrogenases, classified as type $1 \mathrm{~d}, 1 \mathrm{~h}$ and $3 \mathrm{~b}$ using the hydDB classification tool [38]. The type $1 \mathrm{~d}$ and $3 \mathrm{~b}$ hydrogenases are also present in strains SolV and V4, whereas the type $1 \mathrm{~h}$ is only found in the closely related strains Kam1 and SolV (Table 2, Additional file 1: Table S8) $[13,14]$.

The 1d hydrogenase is an oxygen tolerant, membrane attached, uptake hydrogenase encoded by hya $A B C$. In brief, hyaB encodes the catalytic large subunit, hya $A$ encodes an FeS protein with a Tat signal sequence, the small subunit, and hyaC encodes a membrane bound cytochrome B. Electrons are transferred from $\mathrm{HyaB}$ via $\mathrm{HyaB}$ to $\mathrm{HyaC}$ and further to the quinone pool [39]. We suggest that the type 1d hydrogenase is anchored in the membrane and facing the periplasm as depicted in Fig. 4.

The type $3 \mathrm{~b}$ hydrogenase is an oxygen tolerant cytoplasmic NADP dependent hydrogenase encoded 
by $h y h B G S L$. In brief, $h y h S L$ encodes the small and large subunits of the hydrogenase, respectively. The $h y h L$ gene contains a frameshift mutation, that is not present in the draft genome of strain Kam1, and thus may be a sequencing or assembly error [15]. The $h y h B G$ genes encode the electron transfer protein and the catalytic subunit respectively of the predicted NADPH dehydrogenase. It should be noted that the only characterized type $3 \mathrm{~b}$ hydrogenase comes from the archeon Pyrococcus furiosus; it has not yet been tested if the bacterial type $3 \mathrm{~b}$ hydrogenases are NADP dependent [40].

The type $1 \mathrm{~h}$ hydrogenase is an oxygen tolerant high affinity uptake hydrogenase encoded by hhySL coding for the large and small subunit respectively [41].

The gene cluster encoding a type $1 \mathrm{~h} / 5$ type hydrogenase in Mycobacterium smegmatis strain $\mathrm{MC}^{2} 155$ encodes an FeS protein, HhyE, speculated to act as an electron transfer protein linking the hydrogenase to the cells electron transport chain [42]. We did not find a $h h y E$ gene in close proximity to hhySL in strains Kam1, SolV or V4. A blastP search, using MSMEG_2718 coding for HhyE in Mycobacterium smegmatis strain $\mathrm{MC}^{2}$ 155, against the genomes of strains Kam1, SolV and V4 gave no hits, indicating that they do not possess $h h y E$ genes located elsewhere in the genomes.

The products of six genes, hypABCDEF, are necessary for maturation and incorporation of metal cofactors in the active site of NiFe hydrogenases [43]. We found the full hyp $A B C D E F$ gene set in the genomes of strains Kam1, SolV and V4. These are organized as a hypBCDEF gene cluster and a hypA located elsewhere in the genome (Additional file 1: Table S8).

In strains, Kam1 and SolV, hypBCDEF are co-located with the type $1 \mathrm{~h}$ hydrogenase, hhySL, and hypA is located approximately $69 \mathrm{kbp}$ downstream of these. Full genome alignments showed that the genomic region surrounding hypABCDEF is conserved between strains Kam1 and SolV. Aligning the genomes of Strains Kam1 and V4 revealed several rearrangements in the regions harboring hypBCDEF and hypA. The hhySLhypBCDEF genes are located on an approximately $26 \mathrm{kbp}$ genomic region that is conserved between strains Kam1 and SolV. This region is absent from the genome of strain V4, with the exception of hypBCDEF that is found in another part of the genome and encoded on the negative strand (Additional file 1: Figure S8). Similar observations were done for the genomic region encoding hypA (Data not shown). Based on these observations, we speculate that the type $1 \mathrm{~h}$ hydrogenase was present in the last common ancestor of strains Kam1, SolV and V4 and then subsequently lost from strain V4 due to genomic rearrangements.

\section{Nitrogen metabolism}

Strain Kam1 is able to use $\mathrm{N}_{2}$, nitrate and ammonium as nitrogen source [7]. The genomes of strains Kam1, SolV and V4 encode nifHDKENB, the minimum gene set required for nitrogen fixation (Table 2, Additional file 1: Table S9) [13, 44, 45]. In brief, nifHDK encodes the MoFe nitrogenase, and nifENB are needed for assembly and insertion of the FeMoco co-factor [44]. The nitrogenase encoding genes are part of a $29.5 \mathrm{kbp}$ gene cluster, conserved between all three strains. In addition to the nif genes, this gene cluster also encodes fix $A B C X$ and several genes predicted to be involved in regulation and maturation of the nitrogenase. The product of the fix genes couples oxidation of NADH with the simultaneous reduction of high potential quinones and low potential flavodoxins via electron bifurcation. It is believed that this process generates the low potential reductants needed for nitrogen fixation [46]. For strains Kam1 and V4 indirect proof of nitrogen fixation stems from growth in medium without a nitrogen source, whereas nitrogen fixation has been directly demonstrated for strain SolV [3, 7, 47].

Nitrate assimilation to biomass, requires that nitrate is reduced first to nitrite and then further to ammonium, a process requiring a total of eight electrons [48] and references therein.

The genomes of strains Kam1, SolV and V4 encode a nitrate reductase, NasA, and a nitrite reductase nirBD, both predicted to be localized in the cytoplasm (Figs. 4 and 5, Additional file 1: Table S9). In brief, NasA catalyzes the reduction of nitrate to nitrite and NirBD the reduction of nitrite to ammonia.

The nas $A$ and nirBD are found in a nitrogen assimilation gene cluster together with predicted ammonium, nitrate and nitrite transporters. This gene cluster is conserved between all three strains (Additional file 1: Table S9).

The methane monooxygenase, pMMO, and the ammonia monooxygenase, AMO, are highly similar homologous enzymes. In agreement with this, AMO is capable of methane oxidation and pMMO of oxidizing ammonium to hydroxylamine [30, 49]. Hydroxylamine is highly toxic, and must therefore be removed from the cells. Hydroxylamine is oxidized by hydroxylamine oxidoreductase, a process that in ammonia oxidizing bacteria is coupled to energy conservation, but in methanotrophs serves as part of a detoxification mechanism [50]. The genomes of all three strains encode a hydroxylamine oxidoreductase, $\mathrm{HaoAB}$, and a nitric oxide reductase NorBC (Figs. 4 and 5, Additional file 1: Table S9). The traditional view has been that HaoAB catalyzes the four-electron oxidation of hydroxylamine to nitrite. However it has recently been shown that HaoAB catalyzes a three electron oxidation of 


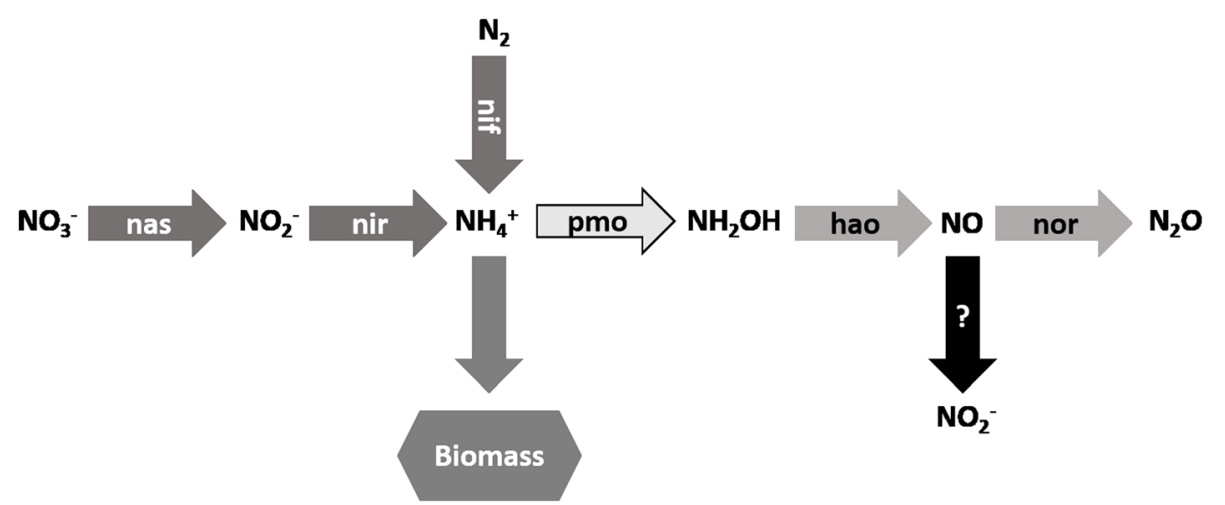

Fig. 5 Overview of the nitrogen metabolism of " $C$. Methylacidiphilum kamchatkense" Kam1. Letters inside arrows refers to the enzymes catalyzing the reaction, see (Additional file 1: Table S9) for locus tags. Dark gray arrows: nitrogen assimilation; light gray arrows: detoxification; white arrow: fortuitous reaction. Nas: assimilatory nitrate reductase; Nir: Nitrite reductase; Nif: nitrogenase; pmo: particulate methane monooxygenase; Hao: hydroxylamine oxidase; Nor: nitric oxide reductase; Black arrow with a question mark: NO oxidation, either nonenzymatic or catalyzed by a yet unidentified NO oxidase

hydroxylamine to nitric oxide [51, 52]. It is therefore likely that they dispose of toxic hydroxylamine by first oxidizing it to nitric oxide that is then reduced to nitrous oxide that diffuses out of the cell (Figs. 4 and 5). However, ammonia oxidation by both methanotrophs and ammonia oxidizing bacteria leads to formation of nitrite (Figs. 4 and 5). The source of the nitrite is still under debate. It has been shown that $\mathrm{NO}$ is oxidized by $\mathrm{O}_{2}$ in a nonenzymatic manner leading to formation of nitrite. However there is evidence that ammonia and methane oxidizing bacteria encodes a hitherto unidentified NO oxidase [51, 52]. The nitrite reductase NirK catalyzing the reduction of nitrite to $\mathrm{NO}$, has been proposed as a possible candidate for this unidentified $\mathrm{NO}$ oxidase, by operating in the reverse direction [51]. Interestingly, strain SolV encodes two putative nitrite reductases (Mfumv2_1120 and 1973). We found a homolog of Mfumv2_1120 in the genome of strain V4 whereas we did not find any nitrite reductases in the genome of strain Kam1 (Additional file 1: Table S9). It has previously been suggested that Mfumv2_1973 encodes a nitrite reductase, NirK, responsible for nitrite reduction in strain SolV, although transcription analysis showed transcription of both Mfumv2_1120 and 1973 [53]. Whole Genome alignments revealed that the genomic regions directly flanking the genes encoding putative nitrite reductases, in strain SolV, are conserved between all three strains (Additional file 1: Figure S8). We performed a blastn search against the NCBI database (17-12-2018) using Mfumv2_1020 and 1973 from strain SolV as query. The best hits for Mfumv2_1120 and 1973 respectively were to sequences from Nitrosomonas eutropha (83\% coverage, $67 \%$ identity) and Oligotropha carboxidovorans (43\% coverage, $67 \%$ identity) and other proteobacteria (Data not shown), indicating that these genes may have been acquired by horizontal gene transfer.

\section{Quinones and respiration complex I to V}

Quinones, are freely diffusible lipophilic electron carriers acting as electron carriers in the membrane [54]. The genome of strain Kam1 does not encode a ubiquinone nor the classical, menFDHCEBIAG, Menaquininone (MK) synthesis pathway [55-57]. Instead, we found genes from the alternative, futalosine, MK synthesis pathway in the genomes of all three strains (Table 2, Additional file 1: Table S10). We were unable to identify a $m q n B$ gene, the product of which is predicted to catalyze the conversion of futalosine or aminodeoxyfutalosine to dehypoxanthinyl futalosine. However, variations of the futalosine pathway are known to exist, it is thus likely that the methylacidiphila encode a yet uncharacterized variant $[58,59]$.

Genes encoding respiration complex I to $\mathrm{V}$ has been identified in "Ca. Methylacidphilum sp." strain RTK17.1 [9]. We found homologs of these in the genomes of strains Kam1, SolV and V4, indicating that they are conserved among methylacidiphila (Table 2, Additional file 1: Table S10). NADH:ubiquinone oxidoreductase (complex I) and succinate dehydrogenase (complex II) represents two entry points of electrons into the quinone pool. The genome of all three strains encodes the classical 14 subunits complex I, consisting of NuoA-N. It is a membrane complex transferring two electrons from $\mathrm{NADH}$ to quinones in the membrane while translocating four protons across the cell membrane, thereby creating a proton gradient [60] and references therein. Complex II, is a cytoplasmic orientated membrane complex encoded by $s d h A B C$. Complex II links the Tricarboxylic acid cycle (TCA) with the respiration chain by transferring two electrons from succinate to the quinone pool, while oxidizing succinate to fumarate [61]. The genome does not encode a complex III, also called $\mathrm{BC}_{1}$ complex, instead it encodes a structurally unrelated but 
functionally similar complex, named alternative complex III (ACIII). This complex transfers electrons from reduced quinones via a cytochrome $\mathrm{c}$ to complex IV, thereby regenerating the quinone pool. It has been proposed that ACIII, also translocates protons across the cell membrane, but this still needs to be experimentally verified $[62,63]$. The genes encoding the ACIII complex is co-located with genes encoding a cbb3 type cytochrome c oxidase (complex IV) (Additional file 1: Table S10). This type of cytochrome c oxidases have very high affinity for oxygen and are often associated with growth under low oxygen tension [64, 65]. This is in line with the observed high oxygen affinity of strain SolV [6], and our observation that strain Kam1 grows optimally at low oxygen concentrations.

The ATP-synthase, complex V, represents the final step in the electron transport chain where the proton gradient generated by the previous steps are consumed to produce ATP.

Previously the presence of two operons encoding different $\mathrm{H}^{+}$translocating F-ATPases were reported from the genome of strain V4 [13]. We found homologs of these operons in the genome of both strains Kam1 and SolV [14]. One is most similar to the F-ATPase found in other verrucomicrobia, whereas the other resembles ATPases found in gamma-proteobacteria [13]. The gene order and content differs between the two operons (Table 2, Additional file 1: Table S10, and Figure S10). The operons encoding the verrucomicrobial and gamma-proteobacterial ATPases is organized as atpBEFHAGDC or atpDCQBEF: $H A G$ respectively (Additional file 1: Figure S10). The organization of the latter resembles that of the $\mathrm{Na}^{+}$translocating N-ATPases, atpDCQRBEFAG, first described by Dibrova and colleagues [66]. N-ATPases are always found in addition to a standard F-ATPase, and are thought to have a role in maintaining cell homeostasis [66]. There are two notable differences between these and the N-ATPase like operon found in strains Kam1, SolV and V4; Firstly, the absence of $a t p R$, which is speculated to interact with the c-subunit [66]. Secondly, the atpE gene, encoding the c-subunit does not encode the $\mathrm{Na}^{+}$binding domain ESTxxY. Recently an $\mathrm{H}^{+}$translocating N-ATPase from the pathogen Burkholderia pseudomallei was characterized. It was suggested that this N-ATPase acts as a highly efficient $\mathrm{H}^{+}$pump, enabling the cells to survive the low $\mathrm{pH}$ inside phagosomes [67]. It is tempting to speculate that in strains Kam1, SolV and V4, the F-ATPase are used for synthesis of ATP, whereas the N-ATPase like ATPase has a role in maintaining cell $\mathrm{pH}$-homeostasis, similarly to what has been suggested for other N-ATPases [66, 67].

\section{Resistance to low $\mathrm{pH}$ and heavy metals}

Acidophiles often have to cope with the dual stress of low $\mathrm{pH}$ and high loads of metals, since metals are more soluble at low $\mathrm{pH}$ [68]. Bacteria can protect themselves against low $\mathrm{pH}$ by passive and active means; the exact mechanism of microbial acid resistance is still not fully understood. Passive mechanisms includes adaptations of the cell membrane making it less permeable for protons and inversion of the membrane potential. Active mechanisms involves removal of protons from the cytoplasm by sequestering and translocation across the inner membrane reviewed in $[69,70]$. Hou and colleagues identified a number of genes with a predicted function in acid resistance in the genome of strain V4 [13]. We identified homologs of these and other genes that may contribute to acid resistance in the genomes of both strains Kam1 and SolV (Additional file 1: Table S11).

The genomes of strains Kam1, SolV and V4 encodes a range of traits that may have a role in maintaining $\mathrm{pH}$ homeostasis of the cell, in addition to the proton translocation steps linked to the activity of respiration complex I to $\mathrm{V}$ discussed above.

We found genes with high homology to gadBC encoding the glutamic acid dependent acid resistance (GDAR) system in Escherichia coli. In brief, $\operatorname{gadB}$ encodes a glutamate decarboxylase catalyzing the proton consuming conversion of glutamate to 4-aminobutanoate and gadC encodes a glutamate/4-aminobutanoate antiporter (for review see [70]). The genomes also encode genes that may have a function analogous to the arginine-dependent acid resistance (ADAR) system in $E$. coli, as suggested for strain V4 [13]. E. coli encodes two arginine decarboxylases, a biosynthetic, speA, and an acid induced adiA. The ADAR system consists of $a d i A$ and an arginine/agmatine antiporter encoded by $a d i C$. The genomes of all three strains encode homologs of speA and adiC, but not adiA (Additional file 1: Table S11).

The genome encodes three genes annotated as either $\mathrm{Na}$-proline symporter or $\mathrm{Na} / \mathrm{H}+$ antiporters in addition to an energy conserving hydrogenase related complex (ehrABCDLS) and a gene, ovp1, encoding a $\mathrm{H}^{+}$-PPase (Additional file 1: Table S11) [71-73]. All of which may have a role in maintaining $\mathrm{pH}$ homeostasis of the cell.

Strain V4 has been reported to encode a mercury resistance system consisting or merRAT:P [13]. The functions are in brief; MerR: transcriptional regulator; MerA: mercury reductase and MerT:P: a fusion protein (Transporter classification database number 1.A.72.3) [74]; MerP is involved in binding mercury in the periplasm before transferring it to MerT that transports mercury across the inner membrane [75]. The mer genes are also found in strains Kam1 and SolV, but in these strains the merT:P and the merRA genes are located at separate places in the genomes (Additional file 1: Table S12).

The genome of all three strains encodes an arsenate resistance gene cluster arsCR-acr3 and an additional orphan $\operatorname{ars} C$ homologue located elsewhere in the genomes. 
The functions are in brief; ars $C$, arsenate reductase; $\operatorname{arsR}$, transcriptional regulator and $a c r 3$, AS (III) efflux pump [76, 77]. Our tests showed that strain Kam1 grows well in the presence of $1 \mathrm{mM}$ As (III) or As(V), but not in the presence of $5 \mathrm{mM}$ of either (Data not shown). Hou and colleagues identified 10 gene clusters encoding TolC, outer membrane proteins and/or AcrA, linking outer and inner membrane channels in addition to genes encoding a putative tellurium and a silver efflux pump (COGs 1538, 0845, 0861 and 3696, respectively) [13, 78]. Our analysis showed that all but one of these gene clusters are conserved between all three strains (Additional file 1: Table S12). We also identified two cation transport ATPases (COG 2217), these are also conserved between all three strains (Additional file 1: Table S12) [78].

\section{Conclusions}

We here present the closed genome of "Ca. Methylacidiphilum kamchatkense" strain Kam1. We used genome analysis to show that strains Kam1 and SolV belongs to two different but closely related species. Our analysis revealed large differences in the numbers of TEs between the three "Ca. Methylacidiphilum" spp. We also found evidence that much of the differences between the three strains can be explained by the action of TEs, exemplified with the loss of a type $1 \mathrm{~h}$ hydrogenase from the genome strain V4 caused by genomic rearrangements. Most of the genes encoding metabolic traits discussed in the present contribution are conserved among all three strains, although they may be found at different places in the genomes due to genomic rearrangements. Finally we present evidence that, $\mathrm{pmoCA}_{4}$ that is unique for strain Kam1, has been present and subsequently lost from the genomes of strains SolV and V4. The availability of three closed genomes have allowed us to do comparative analysis to gain a deeper understanding of the evolution and metabolism of this novel genus. Future studies should focus on linking genome and wet lab experimentation to improve our understanding of their metabolism. It is likely that more genomes from this genus will be available in the future, which will allow further comparative genomics analysis to elucidate even more details of evolution and adaptations to specific niches.

\section{Methods}

\section{Cultivation, DNA extraction and sequencing}

Strain Kam1 were originally isolated from an acid hot spring in Kamchatka, Russia by members of our research group at the University of Bergen, Norway as previously described [7]. The culture used in the present contribution originates from our in house culture collection. It has thus, not been necessary to obtain permission from any third parties, to use this strain in the present study. Strain Kam1 was cultivated at $55^{\circ} \mathrm{C}$ and $\mathrm{pH} 3.5$ with methane as the sole carbon and energy source as described in [10].

Cells were harvested by centrifugation of an early stationary phase culture, before DNA was extracted using the cetyltrimethylammonium bromide method [79].

The genome was sequenced using PacBio technology and assembled with HGAP3 [80], resulting in a single contig of 2,221,048 bp with an average coverage of 489 . Genome sequencing and assembly were done by GATC, Konstanz, Germany. Overlapping ends of the linear contig were manually trimmed as recommended by Chin and colleagues [80], resulting in a final genome size of 2 , 202,032 bp.

\section{Genome annotation and analysis}

Genome annotation and analysis were done using the Integrated Microbial Genomes and Microbiomes IMG/ER platform [17]. Signal sequences were identified with signalp 5.0 [81].

Jspecies were used to calculate Average Nucleotide Identities, [82]. Artemis V16 and Artemis comparison tool V13, were used for visualizing and comparing genomes [83, 84]. Circular genome comparison figures were generated with Blast Ring Image Generator (BRIG) [85]. Dotplots were calculated with Gepard [86]. Protein similarity and identity matrixes were generated with Matrix Global Alignment tool (MATGAT) with default settings [87].

We searched all three genomes for the presence of partial and decayed transposases using the nucleotide sequences of genes annotated as transposases from all three strains as a query for a discontiguous megablast E-10. Insertion sequences were identified with ISsaga www-is.biotoul.fr, predicted sequences flagged as probably false positives were manually curated [88]. Finally, data on annotated transposases, partial transposases and insertion sequences were combined and are for simplicity collectively referred to as transposable elements.

Hydrogenases were classified with HydDB [38]. Local genome searches and alignments were done with Basic Local Alignment Tool (BLAST) [89]. Searches for integrated phages were done using phaster [90]. CRISPRFinder were used to indentify CRISPRs, [91]. Prediction of genomic islands were done using Island Viewer 4 [92]. Five or four genes evenly distributed along the length of Gi-I or II respectively, were selected and used as a query for a blastn search against the nucleotide collection of the NCBI, excluding uncultured organisms. The two best hits, when applicable, were used to get an indication of the phylogenetic association of the GI. 


\section{Additional files}

Additional file 1: Table S1. Average nucleotide identity (ANI); Figure S1. Pairwise synteny dot plots; Figure S2. Organization of CRISPR clusters; Figure S3. Circular representation of the genome of " $\mathrm{C}$. Methylacidiphilum fumariolicum" SolV; Figure S4. Circular representation of the genome of "Ca. Methylacidiphilum infernorum" V4; Table S2. Result of Blastn searches of selected genes from genomic island I and II; Table S3. Genes associated with methane oxidation; Table S4. pmoD homologs; Table S5, A-D). Similarity (bottom) and identity (top) matrix generated from the amino acid sequence of the PmoA, B, C and Ds; Figure S5. Genome alignment of strains Kam1, SolV and V4, showing the region encoding pmo cluster l; Figure S6. Genome alignment of strains Kam1, SolV and V4, showing the region encoding pmo cluster II; Figure S7. Genome alignment of strains Kam1, SolV and V4, showing the region encoding pmo cluster III; Table S6. Genes associated with $\mathrm{CO}_{2}$ fixation; Table S7. Genes associated with glycogen metabolism; Table S8. Genes associated with hydrogenases; Figure S8. Genome alignment of strains Kam1, SolV and V4, showing the region encoding the type $1 \mathrm{~h}$ hydrogenase; Table S9. Genes associated with nitrogen metabolism; Figure S9. Genome alignment of strains Kam1, SolV and V4, showing the regions encoding putative Nirks in strain SolV; Table S10. Genes encoding components of electron transport chains; Figure S10. Organization of the two atp operons; Table S11. Genes speculated to be involved in acid resistance; Table S12. Genes predicted to have a role in heavy metal resistance. (DOCX $3318 \mathrm{~kb}$ )

Additional file 2: Genome coordinates of the features shown on the outer ring of Fig. 1 and Additional file 1: Figures S3-S4. (XLSX 16 kb)

\section{Abbreviations}

ACIII: Alternative complex III; ADAR: Arginine-dependent acid resistance; ANI: Average nucleotide identity; BLAST: Basic Local Alignment Tool; BRIG: Blast Ring Image Generator; Ca.: Candidatus; CBB: Calvin Benson Bassham; Ehr: Ech hydrogenase related; GDAR: Glutamic acid dependent acid resistance; Gl: Genomic island; MATGAT: Matrix Global Alignment tool; MDH: Methanol dehydrogenase; MK: Menaquinone; NCBI: National Center for Biotechnology Information; pMMO: Particulate methane monooxygenase; sMMO: Solouble methane monooxygenase; Tat: Twin arginine translocation; TCA: Tricarboxylic acid cycle; TE: Transposable elements

\section{Acknowledgements}

Not applicable.

\section{Authors' contributions}

TK: Analysed the data, helped in designing laboratory experiments and wrote the manuscript. NKB: Conceived the project, designed the laboratory experiments and wrote the manuscript. CMR and HAE: Planned and performed the laboratory experiments and revised the manuscript. All authors has approved the final manuscript

\section{Funding}

This work was supported by the Research Council of Norway (grant 261923). The funders had no role in study design, data collection and analysis, decision to publish, or preparation of the manuscript.

\section{Availability of data and materials}

The annotated complete genome of "Ca. Methylacidiphilum kamchatkense" Kam1 is deposited at Genbank under accession: CP037899 and at the Integrated Microbial Genomes and Microbiomes IMG/ER server under the genome ID: 2770939480.

\section{Ethics approval and consent to participate}

Not applicable.

\section{Consent for publication}

Not applicable.

\section{Competing interests}

The authors declare that they have no competing interests.
Received: 9 May 2019 Accepted: 26 July 2019

Published online: 09 August 2019

\section{References}

1. Forster P, Ramaswamy V, Artaxo P, Berntsen T, Betts R, Fahey DW, Haywood J, Lean J, Lowe DC, Myhre G et al: Changes in atmospheric constituents and in radiative forcing. In. Edited by Solomon S, Qin D, Manning $M, C$ hen $Z$, Marquis M, Averyt KB, Tignor M, Miller HL. Cambridge: Cambridge University Press; 2007: 129-234.

2. Kalyuzhnaya MG, Puri AW, Lidstrom ME. Metabolic engineering in methanotrophic bacteria. Metab Eng. 2015;29:142-52.

3. Op den Camp HJM, Islam T, Stott MB, Harhangi HR, Hynes A, Schouten S, Jetten MSM, Birkeland N-K, Pol A, Dunfield PF: Environmental, genomic and taxonomic perspectives on methanotrophic Verrucomicrobia. Environ Microbiol Rep 2009, 1(5):293-306.

4. Ettwig KF, Butler MK, Le Paslier D, Pelletier E, Mangenot S, Kuypers MMM, Schreiber F, Dutilh BE, Zedelius J, de Beer D, et al. Nitrite-driven anaerobic methane oxidation by oxygenic bacteria. Nature. 2010;464:543.

5. Dunfield PF, Yuryev A, Senin P, Smirnova AV, Stott MB, Hou S, Ly B, Saw JH, Zhou Z, Ren $Y$, et al. Methane oxidation by an extremely acidophilic bacterium of the phylum Verrucomicrobia. Nature. 2007:450(7171):879-82.

6. Pol A, Heijmans K, Harhangi HR, Tedesco D, Jetten MSM, Op den Camp HJM. Methanotrophy below $\mathrm{pH} 1$ by a new Verrucomicrobia species. Nature. 2007:450:874.

7. Islam T, Jensen S, Reigstad LJ, Larsen O, Birkeland NK. Methane oxidation at 55 degrees $\mathrm{C}$ and $\mathrm{pH} 2$ by a thermoacidophilic bacterium belonging to the Verrucomicrobia phylum. Proc Natl Acad Sci U S A. 2008;105(1):300-4.

8. van Teeseling MCF, Pol A, Harhangi HR, van der Zwart S, Jetten MSM, Op den Camp HJM, van Niftrik L. Expanding the Verrucomicrobial Methanotrophic world: description of three novel species of Methylacidimicrobium gen. Nov. Appl Environ Microbiol. 2014;80(21):6782-91.

9. Carere CR, Hards K, Houghton KM, Power JF, McDonald B, Collet C, Gapes DJ, Sparling R, Boyd ES, Cook GM, et al. Mixotrophy drives niche expansion of verrucomicrobial methanotrophs. Isme j. 2017;11(11):2599-610.

10. Erikstad HA, Jensen S, Keen TJ, Birkeland NK. Differential expression of particulate methane monooxygenase genes in the verrucomicrobial methanotroph 'Methylacidiphilum kamchatkense' Kam1. Extremophiles: Life Under Extreme Conditions. 2012;16(3):405-9.

11. Khadem AF, Pol A, Wieczorek A, Mohammadi SS, Francoijs K-J, Stunnenberg HG, Jetten MSM, Op den Camp HJM. Autotrophic Methanotrophy in Verrucomicrobia: Methylacidiphilum fumariolicum SolV uses the Calvin-BensonBassham cycle for carbon dioxide fixation. J Bacteriol. 2011;193(17):4438-46.

12. Pol A, Barends TRM, Dietl A, Khadem AF, Eygensteyn J, Jetten MSM, Op den Camp HJM. Rare earth metals are essential for methanotrophic life in volcanic mudpots. Environ Microbiol. 2014;16(1):255-64.

13. Hou S, Makarova KS, Saw JH, Senin P, Ly BV, Zhou Z, Ren Y, Wang J, Galperin MY, Omelchenko MV, et al. Complete genome sequence of the extremely acidophilic methanotroph isolate V4, Methylacidiphilum infernorum, a representative of the bacterial phylum Verrucomicrobia. Biol Direct. 2008:3:26.

14. Anvar SY, Frank J, Pol A, Schmitz A, Kraaijeveld K, den Dunnen JT. Op den camp HJ: the genomic landscape of the verrucomicrobial methanotroph Methylacidiphilum fumariolicum SolV. BMC Genomics. 2014;15:914.

15. Erikstad H-A, Birkeland N-K. Draft genome sequence of "Candidatus Methylacidiphilum kamchatkense" strain Kam1, a thermoacidophilic methanotrophic verrucomicrobium. Genome Announc. 2015;3(2):e00065-15. https://doi.org/10.1128/genomeA.00065-15.

16. Mohammadi S, Pol A, van Alen TA, Jetten MS. Op den camp HJ: Methylacidiphilum fumariolicum SolV, a thermoacidophilic 'Knallgas' methanotroph with both an oxygen-sensitive and -insensitive hydrogenase. Isme J. 2017;11(4):945-58.

17. Chen IA, Chu K, Palaniappan K, Pillay M, Ratner A, Huang J, Huntemann M, Varghese N, White JR, Seshadri R, et al. IMG/M v.5.0: an integrated data management and comparative analysis system for microbial genomes and microbiomes. Nucleic Acids Res. 2019;47(D1):D666-d677.

18. Goris J, Konstantinidis KT, Klappenbach JA, Coenye T, Vandamme P, Tiedje JM. DNA-DNA hybridization values and their relationship to whole-genome sequence similarities. Int J Syst Evol Microbiol. 2007;57(Pt 1):81-91.

19. Mohanraju P, Makarova KS, Zetsche B, Zhang F, Koonin EV, van der Oost J. Diverse evolutionary roots and mechanistic variations of the CRISPR-Cas systems. Science (New York, NY). 2016;353(6299):aad5147-1-aad5147-12. 
20. Makarova KS, Wolf YI, Alkhnbashi OS, Costa F, Shah SA, Saunders SJ, Barrangou R, Brouns SJ, Charpentier E, Haft DH, et al. An updated evolutionary classification of CRISPR-Cas systems. Nat Rev Microbiol. 2015; 13(11):722-36

21. Siguier P, Gourbeyre E, Chandler M. Bacterial insertion sequences: their genomic impact and diversity. FEMS Microbiol Rev. 2014;38(5):865-91.

22. Arber W. Genetic variation: molecular mechanisms and impact on microbial evolution. FEMS Microbiol Rev. 2000;24(1):1-7.

23. Luesken FA, Zhu B, van Alen TA, Butler MK, Diaz MR, Song B, Op den Camp HJM, MSM J, Ettwig KF. pmoA primers for detection of anaerobic Methanotrophs. Appl Environ Microbiol. 2011;77(11):3877-80.

24. Ghashghavi M, Jetten MSM, Lüke C. Survey of methanotrophic diversity in various ecosystems by degenerate methane monooxygenase gene primers. AMB Express. 2017;7:162.

25. El Sheikh AF, Poret-Peterson AT, Klotz MG. Characterization of two new genes, $a m o R$ and $a m o D$, in the amo operon of the marine Ammonia oxidizer Nitrosococcus oceani ATCC 19707. Appl Environ Microbiol. 2008; 74(1):312-8

26. Fisher OS, Kenney GE, Ross MO, Ro SY, Lemma BE, Batelu S, Thomas PM, Sosnowski VC, DeHart CJ, Kelleher NL, et al. Characterization of a long overlooked copper protein from methane- and ammonia-oxidizing bacteria. Nat Commun. 2018;9(1):4276.

27. Kenney GE, Sadek M, Rosenzweig AC. Copper-responsive gene expression in the methanotroph Methylosinus trichosporium OB3b. Metallomics: Integrated Biometal Science. 2016;8(9):931-40.

28. Larsen O, Karlsen OA. Transcriptomic profiling of Methylococcus capsulatus (Bath) during growth with two different methane monooxygenases. MicrobiologyOpen. 2016;5(2):254-67.

29. Lawton TJ, Kenney GE, Hurley JD, Rosenzweig AC. The CopC family: structural and Bioinformatic insights into a diverse Group of Periplasmic Copper Binding Proteins. Biochemistry. 2016;55(15):2278-90.

30. Ross MO, Rosenzweig AC. A tale of two methane monooxygenases. J Biol Inorganic Chem. 2017;22(2-3):307-19.

31. Balasubramanian R, Smith SM, Rawat S, Yatsunyk LA, Stemmler TL, Rosenzweig AC. Oxidation of methane by a biological dicopper Centre. Nature. 2010;465(7294):115-9.

32. Ross MO, MacMillan F, Wang J, Nisthal A, Lawton TJ, Olafson BD, Mayo SL, Rosenzweig AC, Hoffman BM. Particulate methane monooxygenase contains only mononuclear copper centers. Science (New York, NY). 2019; 364(6440):566-70.

33. Khadem AF, Pol A, Wieczorek AS, Jetten MS, Op den Camp HJ. Metabolic Regulation of "Ca. Methylacidiphilum Fumariolicum" SolV Cells Grown Under Different Nitrogen and Oxygen Limitations. Frontiers Microbiol. 2012;3:266.

34. Versantvoort W, Pol A, Daumann $\sqcup$, Larrabee JA, Strayer AH, Jetten MSM, van Niftrik L, Reimann J, Op den Camp HJM. Characterization of a novel cytochrome cGJ as the electron acceptor of XoxF-MDH in the thermoacidophilic methanotroph Methylacidiphilum fumariolicum SolV. Biochimica et Biophysica Acta (BBA) - Proteins and Proteomics. 2019;1867(6):595-603.

35. Vorholt JA. Cofactor-dependent pathways of formaldehyde oxidation in methylotrophic bacteria. Arch Microbiol. 2002;178(4):239-49.

36. Khadem AF, van Teeseling MC, van Niftrik L, Jetten MS, Op den Camp HJ, Pol A. Genomic and Physiological Analysis of Carbon Storage in the Verrucomicrobial Methanotroph "Ca. Methylacidiphilum Fumariolicum" SolV. Frontiers Microbiol. 2012;3:345.

37. Chistoserdova L, Chen SW, Lapidus A, Lidstrom ME. Methylotrophy in Methylobacterium extorquens AM1 from a genomic point of view. J Bacteriol. 2003;185(10):2980-7.

38. Søndergaard D, Pedersen CNS, Greening C. HydDB: a web tool for hydrogenase classification and analysis. Sci Rep. 2016;6:34212.

39. Vignais PM, Billoud B. Occurrence, classification, and biological function of hydrogenases: an overview. Chem Rev. 2007;107(10):4206-72.

40. HydDB: A web tool for hydrogenase classification and analysis. 2016. https://services.birc.au.dk/hyddb/browser/class/nife-group-3b/. Acessed 25 Mar 2019.

41. Greening C, Biswas A, Carere CR, Jackson CJ, Taylor MC, Stott MB, Cook GM, Morales SE. Genomic and metagenomic surveys of hydrogenase distribution indicate $\mathrm{H}_{2}$ is a widely utilised energy source for microbial growth and survival. Isme J. 2016;10(3):761-77.

42. Greening C, Constant P, Hards K, Morales SE, Oakeshott JG, Russell RJ, Taylor MC, Berney M, Conrad R, Cook GM. Atmospheric hydrogen scavenging: from enzymes to ecosystems. Appl Environ Microbiol. 2015;81(4):1190-9.
43. Peters JW, Schut GJ, Boyd ES, Mulder DW, Shepard EM, Broderick JB, King PW. Adams MWW: [FeFe]- and [NiFe]-hydrogenase diversity, mechanism, and maturation. Biochimica et Biophysica Acta (BBA) - Molecular Cell Research. 2015;1853(6):1350-69.

44. Dos Santos PC, Fang Z, Mason SW, Setubal JC, Dixon R. Distribution of nitrogen fixation and nitrogenase-like sequences amongst microbial genomes. BMC Genomics. 2012;13(1):162.

45. Khadem AF, Wieczorek AS, Pol A, Vuilleumier S, Harhangi HR, Dunfield PF, Kalyuzhnaya MG, Murrell JC, Francoijs KJ, Stunnenberg HG, et al. Draft genome sequence of the volcano-inhabiting thermoacidophilic methanotroph Methylacidiphilum fumariolicum strain SolV. J Bacteriol. 2012; 194(14):3729-30.

46. Ledbetter RN, Garcia Costas AM, Lubner CE, Mulder DW, TokminaLukaszewska M, Artz JH, Patterson A, Magnuson TS, Jay ZJ, Duan HD, et al. The Electron bifurcating FixABCX protein complex from Azotobacter vinelandii: generation of low-potential reducing equivalents for Nitrogenase catalysis. Biochemistry. 2017;56(32):4177-90.

47. Khadem AF, Pol A, Jetten MS, Op den Camp HJ. Nitrogen fixation by the verrucomicrobial methanotroph 'Methylacidiphilum fumariolicum' SolV. Microbiology (Reading, England). 2010;156(Pt 4):1052-9.

48. Luque-Almagro VM, Gates AJ, Moreno-Vivian C, Ferguson SJ, Richardson DJ, Roldan MD. Bacterial nitrate assimilation: gene distribution and regulation. Biochem Soc Trans. 2011;39(6):1838-43.

49. Hanson RS, Hanson TE. Methanotrophic bacteria. Microbiol Rev. 1996;60(2): 439-71.

50. Stein LY, Klotz MG. Nitrifying and denitrifying pathways of methanotrophic bacteria. Biochem Soc Trans. 2011;39(6):1826-31.

51. Caranto JD, Lancaster KM. Nitric oxide is an obligate bacterial nitrification intermediate produced by hydroxylamine oxidoreductase. Proc Natl Acad Sci. 2017;114(31):8217-22.

52. Versantvoort W, Pol A, Op den Camp HJM, Jetten MSM, Kartal B, Reimann J. A novel hydroxylamine oxidoreductase from a thermoacidophilic volcanic methanotroph for survival of high ammonia stress. Biochimica et Biophysica Acta (BBA) - Bioenergetics. 2018;1859:e118.

53. Mohammadi SS, Pol A, van Alen T, Jetten MSM, Op den Camp HJM. Ammonia oxidation and nitrite reduction in the Verrucomicrobial Methanotroph Methylacidiphilum fumariolicum SolV. Front Microbiol. 2017;8:1901.

54. Simon J, van Spanning RJM, Richardson DJ. The organisation of proton motive and non-proton motive redox loops in prokaryotic respiratory systems. Biochimica et Biophysica Acta (BBA) - Bioenergetics. 2008;1777(12): 1480-90.

55. Meganathan R. Biosynthesis of menaquinone (vitamin K2) and ubiquinone (coenzyme Q): A perspective on enzymatic mechanisms. In: Vitamins \& Hormones. vol. 61. Cambridge: Academic Press; 2001. p. 173-218.

56. Aussel L, Pierrel F, Loiseau L, Lombard M, Fontecave M, Barras F. Biosynthesis and physiology of coenzyme Q in bacteria. Biochimica et Biophysica Acta (BBA) - Bioenergetics. 2014;1837(7):1004-11.

57. Chen M, Ma X, Chen X, Jiang M, Song H, Guo Z. Identification of a hotdog fold Thioesterase involved in the biosynthesis of Menaquinone in Escherichia coli. J Bacteriol. 2013;195(12):2768-75.

58. Arakawa C, Kuratsu M, Furihata K, Hiratsuka T, Itoh N, Seto H, Dairi T. Diversity of the early step of the Futalosine pathway. Antimicrob Agents Chemother. 2011:55(2):913-6.

59. Hiratsuka T, Furihata K, Ishikawa J, Yamashita H, Itoh N, Seto H, Dairi T. An alternative menaquinone biosynthetic pathway operating in microorganisms. Science (New York, NY). 2008;321(5896):1670-3.

60. Berrisford JM, Baradaran R, Sazanov LA. Structure of bacterial respiratory complex I. Biochim Biophys Acta. 2016;1857(7):892-901.

61. Anderson RF, Hille R, Shinde SS, Cecchini G. Electron transfer within complex II: Succinate:ubiquinone oxidoreductase of Escherichia coli. J Biol Chem. 2005;280(39):33331-7.

62. Refojo PN, Teixeira M, Pereira MM. The alternative complex III: properties and possible mechanisms for electron transfer and energy conservation. Biochimica et Biophysica Acta (BBA) - Bioenergetics. 2012;1817(10):1852-9.

63. Sun C, Benlekbir S, Venkatakrishnan P, Wang Y, Hong S, Hosler J, Tajkhorshid E, Rubinstein JL, Gennis RB. Structure of the alternative complex III in a supercomplex with cytochrome oxidase. Nature. 2018;557(7703):123-6.

64. Ducluzeau A-L, Ouchane S, Nitschke W. The cbb3 oxidases are an ancient innovation of the domain Bacteria. Mol Biol Evol. 2008;25(6):1158-66.

65. Pitcher RS, Watmough NJ. The bacterial cytochrome cbb3 oxidases. Biochim Biophys Acta. 2004;1655(1-3):388-99. 
66. Dibrova DV, Galperin MY, Mulkidjanian AY. Characterization of the N ATPase, a distinct, laterally transferred $\mathrm{Na}^{+}$-translocating form of the bacterial F-type membrane ATPase. Bioinformatics (Oxford, England). 2010;26(12):1473-6.

67. Schulz S, Wilkes M, Mills DJ, Kuhlbrandt W, Meier T. Molecular architecture of the N-type ATPase rotor ring from Burkholderia pseudomallei. EMBO Rep. 2017;18(4):526-35

68. Dopson M, Holmes DS. Metal resistance in acidophilic microorganisms and its significance for biotechnologies. Appl Microbiol Biotechnol. 2014;98(19): 8133-44.

69. Baker-Austin C, Dopson M. Life in acid: pH homeostasis in acidophiles. Trends Microbiol. 2007;15(4):165-71.

70. Kanjee U, Houry WA. Mechanisms of acid resistance in Escherichia coli. Annu Rev Microbiol. 2013;67:65-81.

71. Coppi MV. The hydrogenases of Geobacter sulfurreducens: a comparative genomic perspective. Microbiology (Reading, England). 2005;151(Pt 4):1239-54.

72. Marreiros BC, Batista AP, Duarte AMS, Pereira MM. A missing link between complex I and group 4 membrane-bound [NiFe] hydrogenases. Biochimica et Biophysica Acta (BBA) - Bioenergetics. 2013;1827(2):198-209.

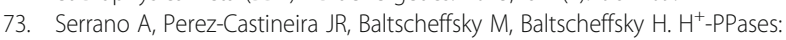
yesterday, today and tomorrow. IUBMB Life. 2007;59(2):76-83.

74. Saier MH Jr, Reddy VS, Tsu BV, Ahmed MS, Li C, Moreno-Hagelsieb G. The transporter classification database (TCDB): recent advances. Nucleic Acids Res. 2016;44(D1):D372-9.

75. Barkay T, Miller SM, Summers AO. Bacterial mercury resistance from atoms to ecosystems. FEMS Microbiol Rev. 2003;27(2-3):355-84.

76. Stolz JF, Basu P, Santini JM, Oremland RS. Arsenic and selenium in microbial metabolism. Annu Rev Microbiol. 2006;60:107-30.

77. Fu HL, Meng Y, Ordonez E, Villadangos AF, Bhattacharjee H, Gil JA, Mateos $L M$, Rosen BP. Properties of arsenite efflux permeases (Acr3) from Alkaliphilus metalliredigens and Corynebacterium glutamicum. J Biol Chem. 2009;284(30):19887-95.

78. Galperin MY, Makarova KS, Wolf YI, Koonin EV. Expanded microbial genome coverage and improved protein family annotation in the COG database. Nucleic Acids Res. 2015;43(Database issue):D261-9.

79. JGI-Bacterial-DNA-isolation-CTAB-Protocol. 2012 https://jgi.doe.gov/userprograms/pmo-overview/protocols-sample-preparation-information/jgibacterial-dna-isolation-ctab-protocol-2012/. Acessed 25 Mar 2019.

80. Chin CS, Alexander DH, Marks P, Klammer AA, Drake J, Heiner C, Clum A, Copeland A, Huddleston J, Eichler EE, et al. Nonhybrid, finished microbial genome assemblies from long-read SMRT sequencing data. Nat Methods. 2013;10(6):563-9.

81. Almagro Armenteros JJ, Tsirigos KD, Sønderby CK, Petersen TN, Winther O, Brunak S, von Heijne G, Nielsen H. SignalP 5.0 improves signal peptide predictions using deep neural networks. Nat Biotechnol. 2019;37(4):420-3.

82. Richter M, Rossello-Mora R. Shifting the genomic gold standard for the prokaryotic species definition. Proc Natl Acad Sci U S A. 2009;106(45):19126-31.

83. Carver TJ, Rutherford KM, Berriman M, Rajandream M-A, Barrell BG, Parkhill J. ACT: the Artemis comparison tool. Bioinformatics (Oxford, England). 2005; 21(16):3422-3.

84. Rutherford K, Parkhill J, Crook J, Horsnell T, Rice P, Rajandream M-A, Barrell B. Artemis: sequence visualization and annotation. Bioinformatics (Oxford, England). 2000;16(10):944-5.

85. Alikhan N-F, Petty NK, Ben Zakour NL, Beatson SA. BLAST ring image generator (BRIG): simple prokaryote genome comparisons. BMC Genomics. 2011;12:402

86. Krumsiek J, Arnold R, Rattei T. Gepard: a rapid and sensitive tool for creating dotplots on genome scale. Bioinformatics (Oxford, England). 2007;23(8):1026-8.

87. Campanella JJ, Bitincka L, Smalley J. MatGAT: an application that generates similarity/identity matrices using protein or DNA sequences. BMC Bioinformatics. 2003;4(1):29.

88. Siguier P, Perochon J, Lestrade L, Mahillon J, Chandler M. ISfinder: the reference Centre for bacterial insertion sequences. Nucleic Acids Res. 2006; 34(Database issue):D32-6.

89. Altschul SF, Gish W, Miller W, Myers EW, Lipman DJ. Basic local alignment search tool. J Mol Biol. 1990;215(3):403-10.

90. Arndt D, Grant JR, Marcu A, Sajed T, Pon A, Liang Y, Wishart DS. PHASTER: a better, faster version of the PHAST phage search tool. Nucleic Acids Res. 2016:44(W1):W16-21.
91. Grissa I, Vergnaud G, Pourcel C. CRISPRFinder: a web tool to identify clustered regularly interspaced short palindromic repeats. Nucleic Acids Res. 2007;35(Web Server issue):W52-7.

92. Bertelli C, Laird MR, Williams KP, Lau BY, Hoad G, Winsor GL, Brinkman FSL. IslandViewer 4: expanded prediction of genomic islands for larger-scale datasets. Nucleic Acids Res. 2017;45(W1):W30-5.

\section{Publisher's Note}

Springer Nature remains neutral with regard to jurisdictional claims in published maps and institutional affiliations.
Ready to submit your research? Choose BMC and benefit from:

- fast, convenient online submission

- thorough peer review by experienced researchers in your field

- rapid publication on acceptance

- support for research data, including large and complex data types

- gold Open Access which fosters wider collaboration and increased citations

- maximum visibility for your research: over $100 \mathrm{M}$ website views per year

At BMC, research is always in progress.

Learn more biomedcentral.com/submissions 